\title{
Quantized vortices in pionic superfluid
}

\author{
Oleg Teryaev ${ }^{1,2.3, *}$ and Valentin Zakharov ${ }^{2,3,4, * *}$ \\ ${ }^{1}$ Joint Institute for Nuclear Research, 141980, Dubna, Russia \\ ${ }^{2}$ Institute of Theoretical and Experimental Physics, NRC Kurchatov Institute, B. Cheremushkinskaya \\ 25, Moscow 117218, Russia \\ ${ }^{3}$ Dubna State University, 141980, Dubna, Russia \\ ${ }^{4}$ Pacific Quantum Center, Far Eastern Federal University, 10 Ajax Bay, Russky Island, Vladivostok \\ 690950, Russia
}

\begin{abstract}
The interplay between classical vorticity being the main undisputed source of polarization in heavy-ion collisions (HIC) and quantized vortices is considered. The vortex tubes emerging in the rotating pionic (super) fluid polarize the baryons in their cores and explain the emerging global polarization. The appearance of vortices in the region separating participants and spectators in non-central HIC is similar to that for sliding layers of liquid helium. From the other side, it is also the region where the classical vorticity was earlier found to be large forming the vortex sheets. The formation of tubes manifests a threshold at certain critical vorticity implying the vanishing polarization at lower energies. For central HIC the compact jet-like flows may lead to formation of vortex rings related to local polarization. The P-odd momentum correlations for their experimental investigation are suggested. The role of shear and viscosity in the emergence of polarization is discussed.
\end{abstract}

\section{Introduction}

Observation of non-vanishing polarization of $\Lambda(\bar{\Lambda})$-hyperons in heavy ion collisions by the STAR Collaboration [1] is considered to be one of most remarkable experimental findings of recent years. The common interpretation nowadays is that spin of the hyperons is aligned with the rotation of plasma produced in peripheral ion-ion collisions. Moreover, such a prediction was made prior to the experimental discovery, (see, e.g. [2-4] and references therein) and explored in some detail after the data appeared [5,6].

The crucial problem is the transition of rotation to spin which may be realized either through the thermal equilibration of spin with rotating matter $[4,5]$ or by the making use $[2,6]$ of axial anomaly in effective theory [7] which naturally led to the predicrion of both polarization decrease with energy [2] and its estimate [3] to be of percent order at several $\mathrm{GeV}$ energy.

The latter approach in confined phase may be realized via the quantum vortices in pionic supeerfluid [8]. The transition of the rotation to spin happens in the cores of these vortices via the heavy baryonic degrees of freedom. This process is accompanied by the dissipation which may be considered as a counterpart if absorptive phases. Such phases are necessary

\footnotetext{
*e-mail: teryaev@jinr.ru

**e-mail: vzakharov@itep.ru
} 
for polarization generation and in non-perturbative QCD describing hadronic processes they are produced by higher twists $[9,10]$ and Wilson lines $[11,12]$.

In the present paper we consider the mechanisms of quantized vortices production in the core-corona border region which was earlier found [13] to produce the femto-vortex sheets ("femto-cyclones") corresponding to classical vorticity.

We relate this phenomenon to the sliding layers in liquid helium considered in classical textbook by R. Feynman [14].

Passing from layer to the compact (mini) jet we generalize this picture to vortex rings being the counterpart of classical ones recently suggested to be produced in pA collisions [15] or by thermalized jets [16].

We suggest the specific spin-monentum correlation to explore such quantized vortex rings. We will also discuss the role of shear and viscosity in polarization emergence.

\section{Sliding layers and quantized vortices}

While both theoretical and exprtimental approaches to liquid helium superfluidity explore the rotating bowl, the situation in HIC is more close to the picture of sliding layers suggested in Feynman's textbook ([14], Chapter 11.9). The relative phase between wave functions of layers results in the extra energy carried by separating surface. It is disappearing in the points where relative phase is proportional to $2 \pi$ leading to the appearance of the vortex lines residing at this boundary and oriented perpendicular to the sliding velocity.

The critical value of this velocity providing the formation of vortices in liquid helium is [14]:

$$
v \geq \frac{1}{m d} L
$$

where $m$ is the mass of helium molecule, $d$ is the transverse size of the region occupied by vortices and $L=\ln d / a$ is the logarithmic factor involving this size and "ultraviolate" (UV) scale provided by interatomic distance $a$.

For HIC the sliding layers correspond to participant and spectator nucleons while $d$ may be related to corona region. $L$ may involve the typical proton radius as a UV scale and is not likely to be large. Assuming the uncertainty relation for $d \sim\left(<p_{T}>\right)^{-1}$ (which will lead to cancellation of $\hbar$ for dimensionfull units) one get the estimate for longitudinal pion momentum $p_{L}=m v$ :

$$
p_{L} \geq \sim<p_{T}>L
$$

Due to the small $L$ this value defined by beam energy is close to the average transverse momentum $<p_{T}>$ of order of $1 \mathrm{GeV}$. This may explain the observed growth of polarization down to $2.4 \mathrm{GeV}$ energy [18]. We may expect that the vortices will not emerge (leading to polarization nullification) only for even lower energies about $1 \mathrm{GeV}$ or so.

As the core-corona region is also the main source of classical vorticity[13], it may be interesting to compare it to the quantum one $\left(\omega_{q}\right)$, produced by the vortex. This procedure is possible as soon as pion fluid viscosity is small albeit different from zero. The expression for critical velocity (1) may be than recasted as an expression for critical vorticity

$$
\omega_{c l} \geq \omega_{q} \sim \frac{1}{\mu d^{2}} L
$$

where we substituted mass by chemical potential (see [8]). 


\section{Vortex rings, local polarization and handedness}

If one pass from consideration of helium flow through long narrow gap (see Fig. 11.32 in [14]), which may correspond to corona region between core and specatators in HIC, to that from the circular one by a sort of (continious) deformation, the vortex lines below and above the gap connect to form the vortex rings.

In HIC this corresponds to the hadronic beams [15] (being most close to the discussed liquid helium example) and jets [16]. One may also consider the rings appearance and evolution in the case of HIC, where 3-fluid hydro [19] or kinetic model [20] can be applied.

While in all these cases the classical vorticity in the rings was considered leading to the local $\Lambda$ polarization, here we suggest to consider the quantized vortex rings. While the result is qualitatively the same (local $\Lambda$ polarization) we offer the another mechanism of its emergence due to excitation of heavy baryonic degrees of freedom in vortex core.

To observe the vortex rings the spin-momentum correlations were suggested in $[15,16]$

$$
R_{\Lambda} \sim<\vec{S}_{\Lambda} \cdot\left[\vec{t} \vec{p}_{\Lambda}\right]>,
$$

involving the $\Lambda$ momentum $\vec{p}_{\Lambda}$ and (average) spin $\vec{S}_{\Lambda}$ as well as trigger vector $\vec{t}$ which can be, in particular, jet trust [16].

Let us first point out that these correlations are the particular case of handedness first suggested just to measure the polarization of quark and gluons fragmenting to hadronic jets [21]. Later it was also applied [22] in the studies of HIC in kinetic model.

Here we wouild like to suggest some modification of this quantity to probe the quantized vortex rings. Let us note that while correlation (5) may indeed signal the apperance of ringlike structure, it may also appear in inclusive polarization, similar to hadronic case [9-12]. Note that this case was actually a guideline in [15] and that inclusive polarization in HIC was recently studied in the course of preparations for experiments at NICA [23].

For more specific probe of quantized vortex formation, we suggest to consider the pion momentum (as forming the ring in the superfluid) instead of that of $\Lambda$ :

$$
R_{\Lambda}^{q} \sim<\vec{S}_{\Lambda} \cdot\left[\vec{t} \vec{p}_{\pi}\right]>
$$

$\vec{t}$ can be either the hadron (or light nucleus, with deuterons being one of the likely first stage beams for NICA [25]) momentum or (mini) jet trust. It is likely that some additional cuts for pion momentum can be applied to select the pions forming the vortex. More studies are definitely required.

\section{Conclusions and outlook}

We suggested here the that production of quantized vortices at the core-corona (and coronaspectators) border in HIC. This phenomenon may be the quantum counterpart of the other roles of this region relevant for baryon polarization [13, 24].

The critical conditions for vortices emergence are discussed and the rough estimate of the minimal energy required for the global polarization generation is performed leading to rather low value $\sim 1 \mathrm{GeV}$.

The formation of vortex rings is considered, the role of handedness in their studies is stressed and its new version is suggested.

Let us also note, that vortex (unlike rigid rotation) produces shear besides vorticity. Indeed, if

$$
v_{i}=k \frac{\epsilon_{i j k} x_{j} s_{k}}{x^{2}},
$$


where $x_{i}$ form the transverse vector w.r.t. polarization $s_{i}$ direction, its derivative

$$
\partial_{l} v_{i}=k\left(\frac{\epsilon_{i l k} s_{k}}{x^{2}}-2 \frac{x_{l} \epsilon_{i j k} x_{j} s_{k}}{\left(x^{2}\right)^{2}}\right)
$$

is not completely antisymmetric in $i, l$. One may guess is there any connection to the much discussed shear-related polarization [26] and explanation of sign puzzle for local polarization.

The shear viscosity plays the crucial role in the transition of rotation to heavy baryon degrees of freedom which is the cornerstone[8] of quantum vortices contribution to polarization. There is also similarity with Kolmogorov cascade with dissipation manifested for smallest vortices.

Finally, let us ask ourselves whether the famous lower bound for viscosity [27] can be related to (upper) bound for polarization?!

\subsection{Acknowledgements}

We are indebted to Yu. Ivanov, M.Lisa and G. Torrieri for discussions.

The work was supported by Russian Science Foundation Grant 21-12-00237.

\section{References}

[1] Global polarization measurement in Au+Au collisions, STAR Collaboration (B.I. Abelev et al.), Phys.Rev. C76 (2007) 024915, Erratum: Phys.Rev. C95 (2017) no.3, 039906 STAR-05-11-2007, e-Print: arXiv:0705.1691 [nucl-ex].

[2] Chiral vortaic effect and neutron asymmetries in heavy-ion collisions O. Rogachevsky, A. Sorin, O. Teryaev, Phys.Rev. C82 (2010) 054910, e-Print: arXiv:1006.1331 [hep-ph] ;

[3] Helicity separation in Heavy-Ion Collisions, M. Baznat, K. Gudima, A. Sorin, O. Teryaev, Phys.Rev. C88 (2013) no.6, 061901 , e-Print: arXiv:1301.7003 [nucl-th];

[4] $\Lambda$ polarization in peripheral heavy ion collisions, F. Becattini, L. Csernai, D.J. Wang, Phys.Rev. C88 (2013) no.3, 034905, Erratum: Phys.Rev. C93 (2016) no.6, 069901, arXiv:1304.4427 [nucl-th];

Angular momentum conservation in heavy ion collisions at very high energy, F. Becattini, F. Piccinini, J. Rizzo, Phys.Rev. C77 (2008) 024906, arXiv:0711.1253 [nucl-th];

F. Becattini, V. Chandra, L. Del Zanna, and E. Grossi, arXiv:1303.3431 [nucl-th].

[5] Global hyperon polarization at local thermodynamic equilibrium with vorticity, magnetic field and feed-down, F. Becattini, I. Karpenko, M. Lisa, I. Upsal, S. Voloshin, e-Print: arXiv:1610.02506 [nucl-th] .

[6] Axial anomaly and energy dependence of hyperon polarization in Heavy-Ion Collisions, A. Sorin, O. Teryaev, Phys.Rev. C95 (2017) no.1, 011902, e-Print: arXiv:1606.08398 [nucl-th] .

[7] A.V. Sadofyev, V.I. Shevchenko, and V.I. Zakharov, Notes on chiral hydrodynamics within effective theory approach, Phys. Rev. D83 (2011) 105025, arXiv:1012.1958 [hepth].

[8] O. V. Teryaev and V. I. Zakharov, Phys. Rev. D 96, no.9, 096023 (2017) doi:10.1103/PhysRevD.96.096023

[9] A. V. Efremov and O. V. Teryaev, Phys. Lett. B 150, 383 (1985) doi:10.1016/03702693(85)90999-2 
[10] J. w. Qiu and G. F. Sterman, Phys. Rev. Lett. 67, 2264-2267 (1991) doi:10.1103/PhysRevLett.67.2264

[11] S. J. Brodsky, D. S. Hwang and I. Schmidt, Phys. Lett. B 530, 99-107 (2002) doi:10.1016/S0370-2693(02)01320-5 [arXiv:hep-ph/0201296 [hep-ph]].

[12] J. C. Collins, Phys. Lett. B 536, $43-48$ (2002) doi:10.1016/S0370-2693(02)01819-1 [arXiv:hep-ph/0204004 [hep-ph]].

[13] M. I. Baznat, K. K. Gudima, A. S. Sorin and O. V. Teryaev, Phys. Rev. C 93, no.3, 031902 (2016) doi:10.1103/PhysRevC.93.031902 [arXiv:1507.04652 [nucl-th]].

[14] R. Feynman, Statistical Mechanics: A Set Of Lectures (W.A. Benjamin, Reading, Mass., 1972)

[15] M. A. Lisa, J. G. P. Barbon, D. D. Chinellato, W. M. Serenone, C. Shen, J. Takahashi and G. Torrieri, Phys. Rev. C 104, no.1, 011901 (2021) doi:10.1103/PhysRevC.104.L011901 [arXiv:2101.10872 [hep-ph]].

[16] W. M. Serenone, J. G. P. Barbon, D. D. Chinellato, M. A. Lisa, C. Shen, J. Takahashi and G. Torrieri, Phys. Lett. B 820, 136500 (2021) doi:10.1016/j.physletb.2021.136500 [arXiv:2102.11919 [hep-ph]].

[17]

[18] F. J. Kornas [HADES], Springer Proc. Phys. 250, 435-439 (2020) doi:10.1007/978-3030-53448-6_68

[19] Y. B. Ivanov and A. A. Soldatov, Phys. Rev. C 97, no.4, 044915 (2018) doi:10.1103/PhysRevC.97.044915 [arXiv:1803.01525 [nucl-th]].

[20] A. Zinchenko, A. Sorin, O. Teryaev and M. Baznat, J. Phys. Conf. Ser. 1435, no.1, 012030 (2020) doi:10.1088/1742-6596/1435/1/012030

[21] A. V. Efremov, L. Mankiewicz and N. A. Tornqvist, Phys. Lett. B 284, 394-400 (1992) doi:10.1016/0370-2693(92)90451-9

[22] O. Teryaev and R. Usubov, Phys. Rev. C 92, no.1, 014906 (2015) doi:10.1103/PhysRevC.92.014906

[23] E. Nazarova, R. Akhat, M. Baznat, O. Teryaev and A. Zinchenko, Phys. Part. Nucl. Lett. 18, no.4, 429-438 (2021) doi:10.1134/S1547477121040142

[24] A. Ayala, M. A. Ayala Torres, E. Cuautle, I. Domínguez, M. A. Fontaine Sanchez, I. Maldonado, E. Moreno-Barbosa, P. A. Nieto-Marín, M. Rodríguez-Cahuantzi and J. Salinas, et al. Phys. Lett. B 810, 135818 (2020) doi:10.1016/j.physletb.2020.135818 [arXiv:2003.13757 [hep-ph]].

[25] A. Arbuzov, A. Bacchetta, M. Butenschoen, F. G. Celiberto, U. D’Alesio, M. Deka, I. Denisenko, M. G. Echevarria, A. Efremov and N. Y. Ivanov, et al. Prog. Part. Nucl. Phys. 119, 103858 (2021) doi:10.1016/j.ppnp.2021.103858 [arXiv:2011.15005 [hep-ex]].

[26] F. Becattini, M. Buzzegoli and A. Palermo, Phys. Lett. B 820, 136519 (2021) doi:10.1016/j.physletb.2021.136519 [arXiv:2103.10917 [nucl-th]].

[27] G. Policastro, D. T. Son and A. O. Starinets, Phys. Rev. Lett. 87, 081601 (2001) doi:10.1103/PhysRevLett.87.081601 [arXiv:hep-th/0104066 [hep-th]]. 